\title{
Rural Spatial Form Based on 3D Simulation Model
}

\author{
Shibo Bi \\ Art College of Xiamen University, Xiamen, China \\ Email:bshibo@163.com
}

How to cite this paper: Bi, S.B. (2019) Rural Spatial Form Based on 3D Simulation Model. Journal of Service Science and Management, 12, 802-809.

https://doi.org/10.4236/jssm.2019.127054

Received: October 10, 2019

Accepted: November 25, 2019

Published: November 28, 2019

Copyright (C) 2019 by author(s) and Scientific Research Publishing Inc. This work is licensed under the Creative Commons Attribution International License (CC BY 4.0).

http://creativecommons.org/licenses/by/4.0/

\begin{abstract}
In order to correlate the rural physical form with the subjective cognition, the rural spatial form and human space behavior were explored. Human cognitive mechanisms in complex spaces were studied. By using virtual reality technology, the spatial morphology and cognitive mechanism of three villages in $\mathrm{X}, \mathrm{Y}$ and $\mathrm{Z}$ were analyzed. The results showed that the three villages had different degrees of spatial perception to the participants. The degree of $X$ was the deepest, the degree of $\mathrm{Y}$ was in the middle, and the degree of $\mathrm{Z}$ was the smallest. This method extends the application of virtual reality technology in the field of traditional village protection. The research results provide theoretical basis and data support for village protection and renewal planning.
\end{abstract}

\section{Keywords}

Rural Area, Spatial Morphological Characteristics, Virtual Reality Technology, Spatial Behavior Characteristics

\section{Introduction}

Most of the traditional villages have gradually developed and perfected. The graceful form of the whole and the spatial structure are formed. In the process of self-organizing growth, site selection, spatial structure and layout of buildings and villages are influenced by multiple factors, such as geographical environment, cultural background, geomantic omen concept, customs, religious etiquette and so on. It has its own spatial morphological features. This result is bound to contain a law and principle of spontaneous organization, as well as the interaction between space form and human behavior. This is the law of space development that needs to be sought [1]. All the cultural and social information in the village space is felt and remembered by people through spatial cognition, which affects the behavior of space use. From the perspective of spatial cogni- 
tion, the village is studied. The user of space is the starting point. The characteristics of the spatial form of the traditional villages are studied. The culture of the village is not closed, but it should be the product of inheritance, creation and continuity. In advocating sustainable development today, the sustainable development of traditional villages is also very important. Therefore, the study of spatial cognition can effectively protect and inherit the traditional villages. Under the new historical conditions, from these traditional villages with great historical value, the positive influence of the current urban development and the construction of the new villages is constantly excavated. In the study of the traditional village space form, the cognition of the village space must be explored. It can better comprehend the culture of the traditional village, and make the historical context better develop and inherit [2].

\section{State of the Art}

The study of Spatial Cognition began in the middle of the twentieth Century. As the main body of space use, human spatial cognition and behavior are not only direct feedback to space form, but also influence the space itself. Therefore, it plays a guiding role in the design. In the study of spatial cognition, the traditional qualitative research method was used in the early stage. The traditional spatial cognition research is based on cognitive psychology. Cognitive map is the main research method. It directly studies the cognition process of people's perception and information acquisition in space [3]. The study of spatial cognition was first derived from cognitive psychology. Cognitive psychology came into being in the middle of the 1950s. It mainly studies the cognitive process of human beings, including attention, perception, imagery, memory, thinking and language. In 1958, British psychologist Donald Broadbent published the book "perception and communication", which laid an important foundation for cognitive psychology. At first, according to the observed phenomena, cognitive psychologists conjectured the psychological process of a person. The nature of the process and the relationship with other processes are determined by measuring the time required for a process. It is called the reaction time method. Later, cognitive psychology began to use experimental, computer simulation, analogy, oral recording and other methods to support the theory of related disciplines. The essential process of human cognitive behavior is studied in depth. Cognitive psychology emphasizes the integrity of the cognitive process. At the same time, the existing knowledge structure in the human brain plays an important role in human behavior and cognition [4] [5].

In space planning and design, art has been widely used in recent years. After the disappearance of the centrality of art, art has gradually evolved into an important medium to shape the characteristics of space. The combination of art and space can create and stimulate people's accidental activities, greatly enrich people's activities and behaviors in space, and bring vitality to the open space.

The space planning under the intervention of art provides an effective way to 
reshape the space characteristics and improve the space quality. 1) Art intervention space planning can break the shackle of the current model design, help culture, create space vitality. 2) Art has become an important medium to shape the characteristics of urban space, and through the communication between man and nature, interaction between man and man, space and time dialogue three ways to achieve its media role. 3) The intervention of art in space planning can make the scattered space layout, accessible space elements, multi-functional space design and extensive public participation, and provide reference for space regeneration and characteristic shaping.

\section{Research Content and Method}

\subsection{Virtual Reality Experiment Platform}

The research adopts virtual reality technology. The technology itself has powerful functional characteristics. Depending on the traditional computer technology, the virtual reality realizes the three-dimensional interaction, which can be well applied to the research of human spatial cognition. The characteristics of virtual reality technology include the following aspects [6]:

Interactivity: The most important feature of virtual reality is interactivity. Through different experience requirements and design effects, the experimenter can freely choose the walking path, control the direction of vision, enter the room, or move objects during the experience. It is different from ordinary $3 \mathrm{D}$ animation [7]. Experience is no longer the single image information. Through subjective cognition, the content and plot in the scene are controlled and influenced. The corresponding feedback is obtained. Compared with the traditional single visual experience mode, the virtual reality can bring the immersive experience of the experimenter to a great extent, which enhances the fun and authenticity of the experiment.

Real time: Another important feature of virtual reality technology is real-time. The rendering of the scene, the input of the signal and the logical judgment of the experiencer are carried out synchronously in the process of the program running. With the rapid development of computer technology, the rendering of high quality scene is realized.

Immersion: Virtual reality technology can provide real experience to the experiencer, and let the experienced people immerse themselves in the virtual environment. In a virtual environment, visual, auditory, tactile and even taste sense can be the same as in the real world. The purpose of virtual reality technology is to provide this immersion for the experiencer [8]. The creation of three-dimensional space and the same behaviors experienced by the experiencer in the virtual environment and the real world, such as walking, running, jumping, sight changing and touching objects, will provide a stronger sense of presence for the experiencer.

As can be seen from Figure 1, virtual reality technology provides richer audio-visual experience for names, which makes people's feelings towards things more intuitive. 

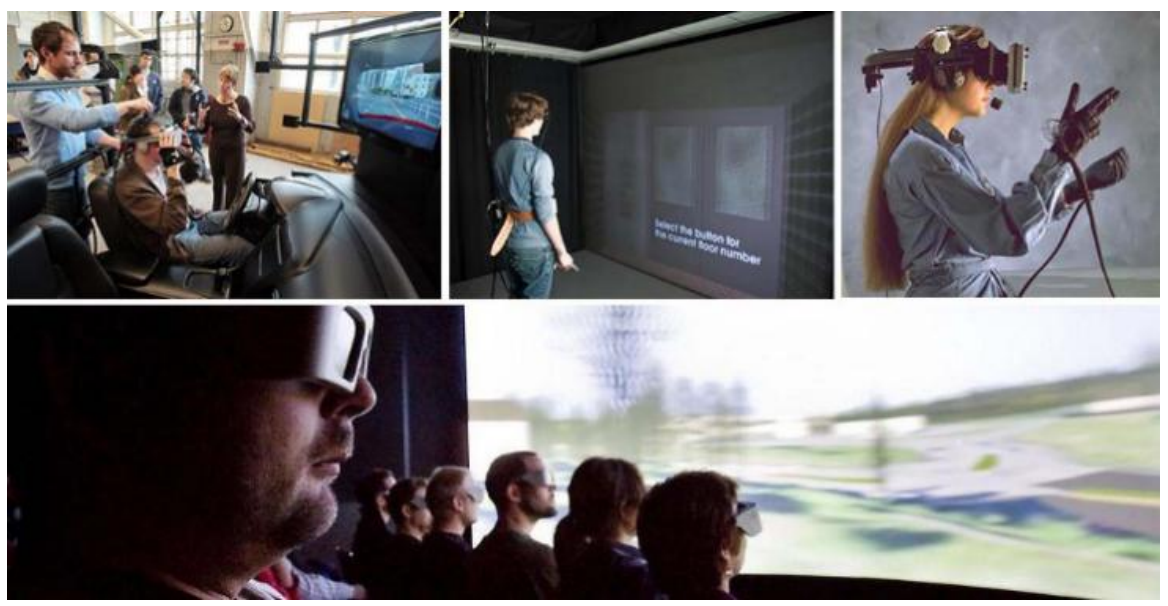

Figure 1. Virtual reality technology.

\subsection{Virtual Scene Modeling}

Before the virtual reality experiment, the most basic work is the environment modeling of the virtual scene. In a real environment, a variety of factors intermingle with each other and act at the same time in the space experience. Therefore, it is difficult to analyze the specific elements in the real environment. In this study, only spatial morphological elements are retained during the establishment of virtual models. This is to ensure that road signs, crowd activities, signs and other non-spatial elements in the real settlement do not affect the path choice of the experiencer in the virtual space roaming process.

In the construction phase of the $3 \mathrm{D}$ scene body model, the modeling software mainly completes the following tasks:

The three-dimensional modeling of environment and building space is almost impossible to achieve in 3D modeling because of Quest 3D. Therefore, all three-dimensional models are dependent on the modeling software. Base form and architectural form of Xidi village is not complicated. In the modeling phase, sketch up software is used. On the basis of general layout and investigation data of Xidi Village, the main model is established.

In the process of modeling, the ground, walls, roofs, doors and windows are grouped in groups based on different materials. It is convenient to import the Autodesk 3ds Max for the texturing adjustment of the next step.

After completing the 3D modeling, in order to achieve the desired visual effect of the material map of the model surface, in the Autodesk 3ds Max software, the model needs to specify the map UV coordinate system.

Using the UVW Mapping modifier in the software, the map type of the object in the model is set to Box. Length, Width, and Height are set to the same appropriate values to ensure that the map is of the same size on the three axes of $\mathrm{X}, \mathrm{Y}$, and $\mathrm{Z}$. At the same time, the position and direction of the map can be adjusted, so that it can be better suited to the model.

Texture baking is a way to store all kinds of information in three-dimensional space, such as illumination, shadow, surface concave-convex and so on. Virtual 
reality uses real-time rendering. This will greatly consume the computing power of CPU, graphics cards, and memory. In order to improve the quality of the picture as much as possible on the premise of guaranteeing the speed, it is necessary to use the method of map baking. Quest-3D can be used to facilitate the application of these baking maps.

\section{Experiment and Result Analysis}

\subsection{Statistical Results}

A total of $52 \mathrm{X}, \mathrm{Y}$, and $\mathrm{Z}$ ancient villages were recruited to investigate the virtual environment experience and space experience. The experience time of each participant was controlled at $7-10$ minutes per village to ensure that the villages had enough time to experience. The participants wore 3D stereoscopic glasses. The operation control device roams the virtual village. In the process of space experience, the path can be freely chosen. The spatial location information was recorded in real time. After the experience of each village, the participants filled out the questionnaire about the village.

According to the spatial characteristics of the ancient villages, 8 adjectives to describe the space are selected by the questionnaire. They are "open space or closed space", "straight road or winding road", "easy identification of space or easy laziness of space", "space mark is obvious or space mark is not obvious", "feel rich in space or monotonous in space," "feel comfortable in space or feel uncomfortable in space," "familiar with space or novel in space." Each pair of adjectives is given a rating scale of five, which is characterized by " $-2,-1,0,1,2$ " respectively.

The evaluation of the three villages is shown in Figure 2. A, B, C, D, E, F, G, $\mathrm{H}$, respectively, is the sense of openness, orientation, smoothness, richness, comfort, clear sense, profound sense, familiarity. Among them, the solid line indicates the evaluation of each village, while the dotted line indicates the overall evaluation.

\subsection{Result Analysis}

As can be seen from Figure 2, the trend of the three evaluation polylines is the same. A sense of direction is the two most left. It showed that the most obvious feelings of the three villages were "the space is easy to get lost" and "the space sign is not obvious".

In the eight evaluations of $\mathrm{X}$, except for the value of "spatial familiarity or spatial novelty" above the overall evaluation value, all other values are below the comprehensive evaluation value. The broken line of $\mathrm{Y}$ is basically coincided with the synthetic evaluation line, and the evaluation line of $\mathrm{Z}$ is on the right side of the comprehensive evaluation line. It shows that the spatial feelings of the three villages are similar on the whole. The space is closed and easy to get lost. The road is tortuous and claustrophous, and the mark is not obvious. Space is more difficult to remember. However, their respective degrees are different. The 


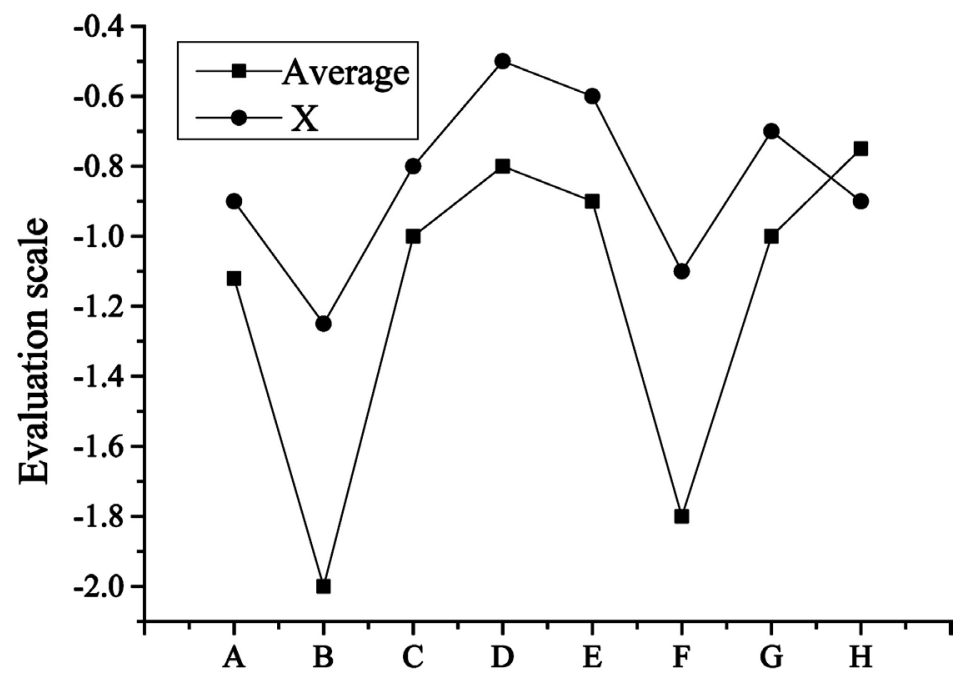

(X)

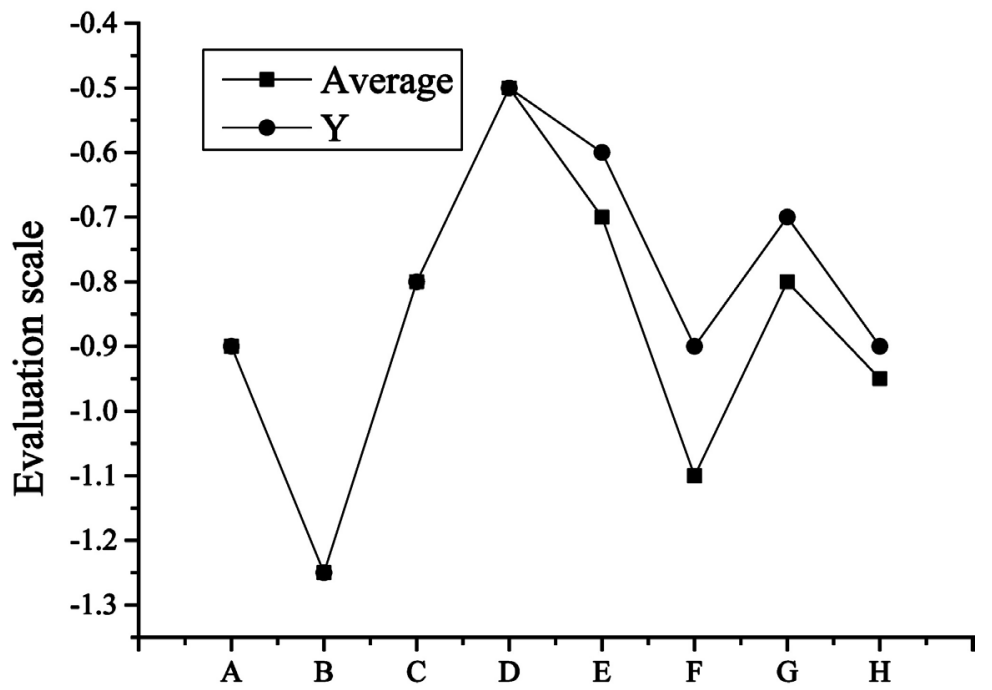

(Y)

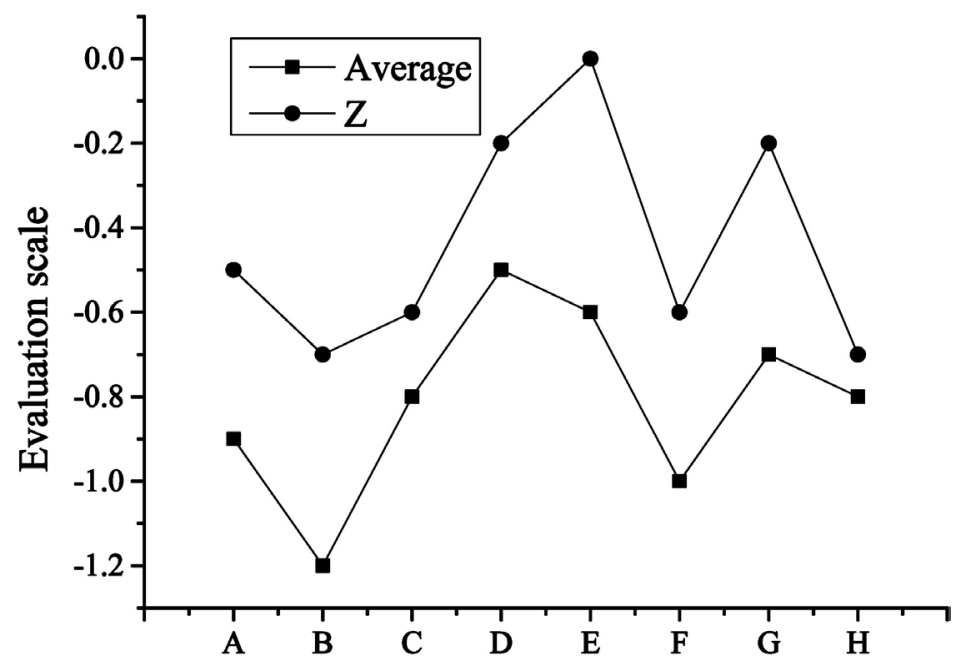

(Z)

Figure 2. Village evaluations of pressure (X, Y, Z). 
degree of $\mathrm{X}$ is the deepest, the degree of $\mathrm{Y}$ is in the middle, and the degree of $\mathrm{Z}$ is the smallest.

\section{Conclusions}

In this paper, virtual reality experiment is combined with quantitative analysis. Through research and experimental analysis, it can be seen that the three villages have certain similarities in cognitive forms. But it also embodies different spatial characteristics. In comparison, the structural clarity of rural roads will greatly affect the distribution of people and the understanding of rural space, with $\mathrm{X}$ being the most obvious. At the same time, the topological characteristics of villages and the density distribution of networks are quite different, so the public's cognition of rural space is fuzzy. $Z$, on the other hand, shows the consistency of topological structure and network density distribution and becomes the most easily recognized space among the three villages.

At present, virtual reality simulation technology is widely used in the field of space simulation, greatly enriched the people's visual experience and sense of space, with the rapid development of science and technology level, believing that the future of virtual reality technology combined with the spatial simulation can bring more surprise, between the limits of science and technology with each passing day and the research of the authors. In this topic research results, there are still some limitations, expecting more in-depth study to make up for in the future.

\section{Conflicts of Interest}

The author declares no conflicts of interest regarding the publication of this paper.

\section{References}

[1] Coxon, M., Kelly, N. and Page, S. (2016) Individual Differences in Virtual Reality: Are Spatial Presence and Spatial Ability Linked. Virtual Reality, 20, 203-212. https://doi.org/10.1007/s10055-016-0292-x

[2] Ricker, B., Schuurman, N. and Kessler, F. (2015) Implications of Smartphone Usage on Privacy and Spatial Cognition: Academic Literature and Public Perceptions. Geojournal, 80, 637-652. https://doi.org/10.1007/s10708-014-9568-4

[3] Gladilin, E. and Eils, R. (2015) On the Role of Spatial Phase and Phase Correlation in Vision, Illusion, and Cognition. Frontiers in Computational Neuroscience, 9, 45. https://doi.org/10.3389/fncom.2015.00045

[4] Wei, T., Cheng, M. and Wenying, F.U. (2015) Reconstruction of Guangzhou Urban Villages' Traditional Lineage Culture in the Context of Rapid Urbanization: From Spatial Form of Ancestral Hall to Behavioral Patterns of Villagers. Acta Geographica Sinica, 70, 1987-2000.

[5] Jiao, H. and Yang, X. (2016) Relationship between Industrial Structure Evolution and Urban Spatial Form Succession of the Coal Resource-Based Cities: A Case Study of Huainan City. Acta Geographica Sinica, 71, 998-1009.

[6] Jiang, H., Zhang, Z. and Dang, J. (2017) Analysis of Geometric Multibounced Vir- 
tual Scattering Channel Model for Dense Urban Street Environments. IEEE Transactions on Vehicular Technology, 66, 1903-1912.

https://doi.org/10.1109/TVT.2016.2574925

[7] Dolins, F.L., Schweller, K. and Milne, S. (2017) Technology Advancing the Study of Animal Cognition: Using Virtual Reality to Present Virtually Simulated Environments to Investigate Nonhuman Primate Spatial Cognition. Current Zoology, 63, 97-108. https://doi.org/10.1093/cz/zow121

[8] Hardcastle, K., Ganguli, S. and Giocomo, L.M. (2017) Cell Types for Our Sense of Location: Where We Are and Where We Are Going. Nature Neuroscience, 20, 1474. https://doi.org/10.1038/nn.4654 